\title{
Three-dimensional assessment of mandibular and glenoid fossa changes after bone-anchored Class III intermaxillary traction
}

\author{
Hugo De Clerck ${ }^{a}$, Tung Nguyen ${ }^{b}$, Leonardo Koerich de Paulac, and Lucia Cevidanes ${ }^{d}$ \\ ${ }^{a}$ Adjunct professor, Department of Orthodontics, School of Dentistry, University of North Carolina, \\ Chapel Hill; private practice, Brussels, Belgium \\ ${ }^{b}$ Assistant professor, Department of Orthodontics, University of North Carolina, Chapel Hill \\ 'Graduate student, Department of Orthodontics and Pediatric Dentistry, Federal University of Rio \\ de Janeiro, Rio de Janeiro, Brazil \\ ${ }^{\mathrm{d} A s s i s t a n t}$ professor, Department of Orthodontics and Pediatric Dentistry, University of Michigan, \\ Ann Arbor
}

\begin{abstract}
Introduction-Conventional treatment for young Class III patients involves extraoral devices designed to either protract the maxilla or restrain mandibular growth. The use of skeletal anchorage offers a promising alternative to obtain orthopedic results with fewer dental compensations. Our aim was to evaluate 3-dimensional changes in the mandibles and the glenoid fossae of Class III patients treated with bone-anchored maxillary protraction.
\end{abstract}

Methods-Twenty-five consecutive skeletal Class III patients between the ages of 9 and 13 years (mean age, $11.10 \pm 1.1$ year) were treated with Class III intermaxillary elastics and bilateral miniplates ( 2 in the infrazygomatic crests of the maxilla and 2 in the anterior mandible). The patients had cone-beam computed tomography images taken before initial loading and at the end of active treatment. Three-dimensional models were generated from these images, registered on the anterior cranial base, and analyzed by using color maps.

Results-Posterior displacement of the mandible at the end of treatment was observed in all subjects (posterior ramus: mean, $2.74 \pm 1.36 \mathrm{~mm}$; condyles: mean, $2.07 \pm 1.16 \mathrm{~mm}$; chin: mean, $-0.13 \pm 2.89 \mathrm{~mm}$ ). Remodeling of the glenoid fossa at the anterior eminence (mean, $1.38 \pm 1.03$ $\mathrm{mm}$ ) and bone resorption at the posterior wall (mean, $-1.34 \pm 0.6 \mathrm{~mm}$ ) were observed in most patients.

Conclusions-This new treatment approach offers a promising alternative to restrain mandibular growth for Class III patients with a component of mandibular prognathism or to compensate for maxillary deficiency in patients with hypoplasia of the midface. Future studies with long-term follow-up and comparisons with facemask and chincup therapies are needed to better understand the treatment effects.

Class III malocclusion is most commonly associated with hypoplasia of maxillary growth. However, hyperplasia of the mandible also can result in a mesiocclusion. Even when maxillary deficiency is the main etiology of the Class III malocclusion, a mandibular retraction effect can aid in the correction of the maxillomandibular discrepancy.

Copyright (C) 2012 by the American Association of Orthodontists.

Reprint requests to: Hugo De Clerck, Kerkstraat 120, 1150 Brussels, Belgium; hugo.declerck@skynet.be.

The authors report no commercial, proprietary, or financial interest in the products or companies described in this article. 
Whereas extraoral traction with a facemask applies anteriorly directed forces to the maxilla to mechanically disrupt the sutures and to stimulate maxillary forward growth, reaction forces tend to push the chin posteriorly. ${ }^{1-4}$ These reaction forces result in clockwise rotation of the mandible and increased vertical dimensions $s^{2,3,5}$ and are also observed in chincup therapy. ${ }^{6,7}$ Little is known about eventual modifications in the temporomandibular joint with facemask therapy. Modeling of the glenoid fossa has been reported in magnetic resonance imaging studies with the Herbst appliance ${ }^{8}$ and in histologic studies on rhesus monkeys treated with chincup therapy. ${ }^{9}$ However, there are no reported human studies of modeling of the glenoid fossa after Class III extraoral traction. Furthermore, 2-dimensional cephalometric analysis does not allow clear visualization of changes in the glenoid fossa or asymmetric effects on both sides.

In contrast to facemask therapy, bone-anchored maxillary protraction applies continuous anteriorly directed forces to the maxilla and continuous retraction forces to the mandible. ${ }^{10,11}$ Moreover, better compliance can be expected from patients for intraoral elastic traction rather than an extraoral device. Previous studies have evaluated the effects of this protocol on maxillary displacement and modeling by using superimpositions of conebeam computed tomography (CBCT) images before and after orthopedic traction registered on the anterior cranial base. ${ }^{12-14}$ In this study, we focused on the mandibular and glenoid fossa changes in 3 dimensions after bone-supported continuous Class III intermaxillary traction.

\section{MATERIAL AND METHODS}

The prospective sample consisted of 25 consecutively treated patients (13 girls, 12 boys) with a dentoskeletal Class III malocclusion. All patients were treated by 1 operator (H.D.C.) with the bone-anchored maxillary protraction technique. Institutional review board approval was obtained before the study.

At the initial observation (T1), all patients had a Class III malocclusion in the mixed or permanent dentition characterized by a Wits appraisal of $-1 \mathrm{~mm}$ or less (mean, $-4.8 \pm 2.8$ $\mathrm{mm}$ ), an anterior crossbite or incisor end-to-end relationship, and a Class III molar relationship. The skeletal characteristics of this sample at $\mathrm{T} 1$ have been previously described in detail. ${ }^{15}$ All patients were of white ancestry, with a prepubertal stage of skeletal maturity according to the cervical vertebral maturation method (CS 1 to CS 3). ${ }^{16}$ Twenty-one of the 25 patients were still prepubertal at the end of treatment (T2) (CS 1 to CS 3), whereas 4 patients were at CS 4 . The subjects' mean ages were $11.9 \pm 1.8$ years at T1 and $13.1 \pm 1.7$ years at $\mathrm{T} 2$. The mean duration of the treatment was $1.2 \pm 0.1$ years.

The bone-anchored maxillary protraction orthopedic protocol consisted of the following. Each patient had miniplates placed on the left and right infrazygomatic crests of the maxillary buttress and between the lower left and right lateral incisors and canines (Fig 1). Small mucoperiosteal flaps were elevated, and modified miniplates (Tita-Link; Bollard, Brussels, Belgium) were secured to the bone by 2 screws in the mandible or 3 screws in the maxilla screw diameter, $2.3 \mathrm{~mm}$; length, $5 \mathrm{~mm}$ ). ${ }^{10}$ The extensions of the plates perforated the attached gingiva near the mucogingival junction. Three weeks after surgery, the miniplates were loaded. Class III elastics applied an initial force of $150 \mathrm{~g}$ on each side, and increased to $200 \mathrm{~g}$ after 1 month of traction and to $250 \mathrm{~g}$ after 3 months. The patients were asked to replace the elastics at least once a day and wear them 24 hours per day. In 14 patients, after 2 to 3 months of intermaxillary traction, a removable biteplate was inserted in the maxillary arch to eliminate occlusal interference in the incisor region until correction of the anterior crossbite was obtained. 
The CBCT scans were taken at T1 and T2 with an iCat machine (Imaging Sciences International, Hatfield, $\mathrm{Pa}$ ) with a $16 \times 22$-cm field of view. Virtual 3-dimensional surface models were constructed from the CBCT images with a voxel dimension of $0.5 \times 0.5 \times 0.5 \mathrm{~mm}$. Construction of 3-dimensional surface models of the anatomic structures of interest and the 3-dimensional graphic renderings were done by using software (ITK-SNAP; open-source software, www.itksnap.org).

The $\mathrm{T} 1$ and T2 images were registered by using the anterior cranial fossa as the reference. A fully automated voxel-wise rigid registration method was performed with software (IMAGINE, open-source software, http://www.ia.unc.edu/dev/download/imagine/ index.htm). This method, developed by Cevidanes et al, ${ }^{17}$ masks anatomic structures altered by treatment or growth to prevent observer-dependent reliance on subjectively defined anatomic landmarks. In this study, the 3-dimensional models at T1 and T2 were registered on anterior cranial fossa structures, specifically the endocranial surfaces of the cribriform plate region of the ethmoid bone and the frontal bone. These regions were chosen because of their early completion of growth. The software compares the images by using the intensity of the gray scale for each voxel of the 2 images.

After the registration step, all reoriented virtual models were superimposed to quantitatively evaluate the greatest surface displacement by using the CranioMaxilloFacial (CMF) application software (developed at the M. E. Müller Institute for Surgical Technology and Biomechanics, University of Bern, Bern, Switzerland, under the funding of the Computer Aided and Image Guided Medical Interventions network, http://co-me.ch). The CMF tool calculates thousands of color-coded point-to-point comparisons (surface distances in millimeters) between the 3 -dimensional models, so that the differences between 2 surfaces at any location can be quantified.

For quantitative assessment of the changes between the 3-dimensional surface models, the isoline tool allows the user to define a surface-distance value to be expressed as a contour line (isoline) that corresponds to regions having a surface distance equal to or greater than the defined value. The isoline tool was used to quantitatively measure the greatest displacements between points in the 3-dimensional surface models for the right and left anterior and posterior surfaces of the condyles, the right and left anterior and posterior surfaces of the glenoid fossae walls, the chin, the right and left posterior surfaces of the rami, and the soft-tissue chin (Fig 2).

The greatest displacements between $\mathrm{T} 1$ and $\mathrm{T} 2$ were computed at each anatomic region of interest. The error of the method as determined in previous studies showed excellent reliability. ${ }^{12}$ Descriptive statistics was used to describe the percentiles, means, standard deviations, and ranges. Pearson correlation coefficients were used to assess the associations between changes at each anatomic region. The level of significance was set at 0.05 .

\section{RESULTS}

Table I summarizes the descriptive statistics for changes in the mandibular condyle, glenoid fossa, ramus, and chin observed from $\mathrm{T} 1$ to $\mathrm{T} 2$ for the 25 consecutive patients treated with bone-anchored maxillary protraction.

Throughout a year of treatment, the chin on average maintained its relative anteroposterior position (mean change, $-0.13 \pm 2.89 \mathrm{~mm}$ ). The range of response was highly variable, from $4.42 \mathrm{~mm}$ of anterior displacement to as much as $-5.85 \mathrm{~mm}$ of posterior displacement. On average, distal displacement of the posterior ramus was observed (mean right ramus displacement, $2.73 \pm 1.36 \mathrm{~mm}$; mean left ramus displacement, $2.76 \pm 1.36 \mathrm{~mm}$ ) (Fig 3). The condyles moved posteriorly (mean right condyle posterior surface changes, $2.03 \pm 1.21 \mathrm{~mm}$; 
mean left condyle posterior surface changes, $2.12 \pm 1.06 \mathrm{~mm}$ ). The posterior eminence of the glenoid fossae showed resorption of the posterior wall (mean right posterior wall changes, $-1.39 \pm 0.75 \mathrm{~mm}$; mean left posterior wall changes, $-1.30 \pm 0.46 \mathrm{~mm}$ ). There was apposition of bone at the anterior eminences of the fossae, with mean changes of $1.30 \pm 1.03$ $\mathrm{mm}$ and $1.47 \pm 1.03 \mathrm{~mm}$, respectively, at the right and left anterior articular eminences (Fig 4). The soft-tissue chin (mean displacement, $-0.03 \pm 3.0 \mathrm{~mm}$ ) showed similar positional changes compared with the hard-tissue chin $(-0.13 \pm 2.89 \mathrm{~mm})$.

Table II shows the correlations between changes at the condyles, glenoid fossae, posterior surface of the rami, and hard- and soft-tissue chins. The amounts of displacement of the right condyle, glenoid fossa, and ramus were highly correlated to the displacements of the same anatomic regions on the left side. There also was a high correlation between the posterior displacement of the condyles and the amount of bone apposition at the anterior eminence and resorption at the posterior wall of the glenoid fossa. Poor or no correlation was found between the displacement of the posterior border of the ramus and the displacement of the condyles or glenoid fossa modeling. However, the anteroposterior displacement of the hard-tissue chin was correlated with the displacement of the posterior border of the ramus and strongly correlated with the displacement of the soft-tissue chin.

\section{DISCUSSION}

Bone-anchored maxillary protraction treatment resulted in the maintenance of the anteroposterior position of the chin $(-0.13 \mathrm{~mm})$. Our 2-dimensional study comparing boneanchored maxillary protraction with untreated Class III controls showed a similar mean value for mandibular restraint, whereas the untreated Class III subjects had $2.2 \mathrm{~mm}$ of anterior displacement of the chin during the same time span. ${ }^{11}$ This contributed to $40 \%$ of the overall improvement in the maxillomandibular discrepancy as expressed by the Wits appraisal. The large variability of change in the anteroposterior position of the chin can be explained by a combination of 4 separate factors that occur simultaneously to determine the individual mandibular pattern of growth and response to treatment: (1) the amount and direction of condylar growth, (2) bone remodeling in the articular fossa, (3) clockwise or counterclockwise rotation of the mandible (matrix rotation), ${ }^{18}$ and (4) the amount of closure or opening of the gonial angle (intramatrix rotation). ${ }^{18}$

As reported in a previous 2-dimensional study, an increase in mandibular length of about 3 $\mathrm{mm}$ per year can be expected in an untreated Class III population of the same age as the sample in our study. ${ }^{19}$ Our 2-dimensional comparison of bone-anchored maxillary protraction and untreated controls showed no significant differences in the changes of mandibular body length and ramus height; ie, the amounts of mandibular growth observed in the bone-anchored maxillary protraction and the untreated samples were similar. ${ }^{11}$ The 2.7 $\mathrm{mm}$ difference in the forward projection of the chin between the bone-anchored maxillary protraction and the untreated controls could not be explained by the restricted growth of the mandible. In another study on the effects of chincup therapy, the increase of the length of the mandible was almost the same as in the control group. ${ }^{20}$

The superimposition and semitransparent overlays in this study indicate that bone-anchored maxillary protraction growth and treatment response result in bone apposition at the anterior eminence of the temporomandibular joint; this correlates well with the posterior displacement of the anterior surface of the condyle, and the bone resorption of the posterior wall of the articular eminence correlates well with the posterior displacement of the posterior surface of the condyle. The high correlation between modeling at the anterior and posterior eminences of the glenoid fossa and the displacement of the opposed surfaces of the condyle indicate that the anteroposterior displacement of the chin was not due to a positional 
shift of the mandible between $\mathrm{T} 1$ and $\mathrm{T} 2$, after correction of the anterior crossbite. The posterior displacement of the condyles occurred while maintaining condylar width. Superimposition of the 2 CBCT images of growing patients, registered on the anterior cranial base, makes it possible to visualize the regions of apposition and resorption in the left and right glenoid fossae. However, this surface modeling is the result of a combination of normal growth and the effect of the orthopedic traction. Only when the CBCT images of untreated Class III subjects are available will the impact of bone-anchored maxillary protraction on the overall modification of the fossae be known. Although the modeling processes in the temporomandibular joint might contribute to posterior displacement (relocation) of the whole mandible, the magnitude of these changes measured in this study was too small to explain entirely the restriction of the forward projection of the chin. Moreover, no correlation was found between chin displacement and condylar changes or fossa modeling. Another interesting illustration of the relationship between condylar changes and glenoid fossa remodeling is in the correction of a Class III malocclusion with mandibular asymmetry with unilateral elastic traction (Fig 5).

Posterior rotation of the mandible also results in posterior displacement of the chin. In our 2dimensional study, such a clockwise rotation was observed in the untreated Class III sample; however, the mean mandibular plane angle in the bone-anchored maxillary protraction group slightly decreased, indicating a mean counter-clockwise rotation. ${ }^{11}$ In another 2dimensional study comparing the outcome of the bone-anchored maxillary protraction protocol with a matched facemask group, the mandibular line showed a significantly different rotation in relation to both the cranial base and the nasal line, with a slight mandibular counterclockwise rotation with bone-anchored maxillary protraction compared with clockwise rotation with the facemask. ${ }^{15}$ In the 3-dimensional overlay of our sample, parallel lowering without rotation of the mandibular border was commonly observed.

In this study, the posterior borders of the left and right mandibular rami were displaced over a mean distance of about $2.7 \mathrm{~mm}$. Anteroposterior changes in the position of the chin were correlated with the ramus displacement. The posterior displacement of the posterior surface of the ramus was greater than the displacement of the condyles, which could be visualized on the semitransparency overlays as a slight swing-back of the ramus (Fig 6). Posterior displacement of the gonial landmarks was also confirmed by the thin-plate spline analysis of our sample. ${ }^{21}$ This displacement without clockwise rotation of the mandible must result in a reduction of the gonial angle. This was confirmed by a decrease of $4.1^{\circ}$ of the gonial angle compared with the control group in our 2-dimensional study. ${ }^{11}$ Similar changes in mandibular shape have been also reported in animal experiments ${ }^{22}$ and in a sample of growing children with mandibular prognathism treated by chincup therapy. ${ }^{23}$

Although previous studies have shown that the treatment protocol applied in this study is indicated for protraction of the midface, this study additionally demonstrates that compensatory effects on mandibular growth considerably contribute to correcting the skeletal discrepancy and improving the facial profile. Three-dimensional imaging makes it possible to better understand the morphologic changes and facilitates assessment of the long-term results in the future.

\section{CONCLUSIONS}

Previous studies have shown that bone-anchored maxillary protraction stimulates forward displacement and modeling of the maxillary and zygoma bones as 1 unit. This study also shows that mandibular shape rather than mandibular size is affected by continuous intermaxillary traction. Moreover, 3-dimensional imaging makes it possible to visualize the 
modeling processes in the glenoid fossae and condyles. Based on the findings of this study, the following can be concluded.

1. Restriction of forward displacement of the chin can be obtained by a combination of a slight swing-back of the ramus and closure of the gonial angle.

2. There was no posterior rotation of the mandible or no temporary positional shift.

3. There was a high correlation between modeling of the anterior and posterior eminences of the glenoid fossae and displacement of the opposing condylar surfaces.

4. There was interindividual variability in treatment outcomes.

\section{References}

1. Pangrazio-Kulbersh V, Berger J, Kersten G. Effects of protraction mechanics on the midface. Am J Orthod Dentofacial Orthop. 1998; 114:484-91. [PubMed: 9810043]

2. Hata S, Itoh T, Nakagawa M, Kamagashira K, Ichikawa K, Matsumoto M, et al. Biomechanical effects of maxillary protraction on the craniofacial complex. Am J Orthod Dentofacial Orthop. 1987; 91:305-11. [PubMed: 3471073]

3. Chong YH, Ive JC, Årtun J. Changes following the use of protraction headgear for early correction of Class III malocclusion. Angle Orthod. 1996; 66:351-62. [PubMed: 8893105]

4. Westwood PV, McNamara JA Jr, Baccetti T, Franchi L, Sarver DM. Long-term effects of Class III treatment with rapid maxillary expansion and facemask therapy followed by fixed appliances. Am J Orthod Dentofacial Orthop. 2003; 123:306-20. [PubMed: 12637903]

5. Baik HS. Clinical results of the maxillary protraction in Korean children. Am J Orthod Dentofacial Orthop. 1995; 108:583-92. [PubMed: 7503035]

6. Deguchi T, McNamara JA Jr. Craniofacial adaptations induced by chincup therapy in Class III patients. Am J Orthod Dentofacial Orthop. 1999; 115:175-82. [PubMed: 9971929]

7. Lin HC, Chang HP, Chang HF. Treatment effects of occipitomental anchorage appliance of maxillary protraction combined with chincup traction in children with Class III malocclusion. $\mathbf{J}$ Formos Med Assoc. 2007; 106:380-91. [PubMed: 17561473]

8. Ruf S, Pancherz H. Temporomandibular joint remodeling in adolescents and young adults during Herbst treatment: a prospective longitudinal magnetic resonance imaging and cephalometric radiographic investigation. Am J Orthod Dentofacial Orthop. 1999; 115:607-18. [PubMed: 10358242]

9. Janzen EK, Bluher JA. The cephalometric, anatomic, and histologic changes in Macaca mulatta after application of a continuous-acting retraction force on the mandible. Am J Orthod. 1965; 51:823-55. [PubMed: 4953378]

10. De Clerck HJ, Cornelis MA, Cevidanes LH, Heymann GC, Tulloch CJ. Orthopedic traction of the maxilla with miniplates: a new perspective for treatment of midface deficiency. J Oral Maxillofac Surg. 2009; 67:2123-9. [PubMed: 19761906]

11. De Clerck H, Cevidanes L, Baccetti T. Dentofacial effects of bone-anchored maxillary protraction: a controlled study of consecutively treated Class III patients. Am J Orthod Dentofacial Orthop. 2010; 138:577-81. [PubMed: 21055597]

12. Cevidanes LH, Heymann G, Cornelis MA, De Clerck HJ, Tulloch JF. Superimposition of 3dimensional cone-beam computed tomography models of growing patients. Am J Orthod Dentofacial Orthop. 2009; 136:94-9. [PubMed: 19577154]

13. Heymann GC, Cevidanes L, Cornelis M, De Clerck HJ, Tulloch JF. Three-dimensional analysis of maxillary protraction with inter-maxillary elastics to miniplates. Am J Orthod Dentofacial Orthop. 2010; 137:274-84. [PubMed: 20152686]

14. Nguyen T, Cevidanes L, Cornelis MA, Heymann G, de Paula LK, De Clerck H. Three-dimensional assessment of maxillary changes associated with bone anchored maxillary protraction. Am J Orthod Dentofacial Orthop. 2011; 140:790-8. [PubMed: 22133943] 
15. Cevidanes L, Baccetti T, Franchi L, McNamara JA Jr, De Clerck H. Comparison of two protocols for maxillary protraction: bone anchors versus face mask with rapid maxillary expansion. Angle Orthod. 2010; 80:799-806. [PubMed: 20578848]

16. Baccetti T, Franchi L, McNamara JA Jr. An improved version of the cervical vertebral maturation (CVM) method for the assessment of mandibular growth. Angle Orthod. 2002; 72:316-23. [PubMed: 12169031]

17. Cevidanes LH, Styner MA, Proffit WR. Image analysis and superimposition of 3-dimensional cone-beam computed tomography models. Am J Orthod Dentofacial Orthop. 2006; 129:611-8. [PubMed: 16679201]

18. Björk A, Skieller V. Normal and abnormal growth of the mandible. A synthesis of longitudinal cephalometric implant studies over a period of 25 years. Eur J Orthod. 1983; 5:1-46. [PubMed: 6572593]

19. Alexander AE, McNamara JA Jr, Franchi L, Baccetti T. Semilongitudinal cephalometric study of craniofacial growth in untreated Class III malocclusion. Am J Orthod Dentofacial Orthop. 2009; 135:700.e1-14. [PubMed: 19524825]

20. Mimura H, Deguchi T. Morphologic adaptation of temporomandibular joint after chincup therapy. Am J Orthod Dentofacial Orthop. 1996; 110:541-6. [PubMed: 8922514]

21. Baccetti T, De Clerck HJ, Cevidanes LH, Franchi L. Morphometric analysis of treatment effects of bone-anchored maxillary protraction in growing Class III patients. Eur J Orthod. 2011; 33:121-5. [PubMed: 21187527]

22. Kanematsu S. Dentofacial changes produced by extraoral posterior force on the mandible of Macaca irus. J Jpn Orthod Soc. 1988; 47:1-36.

23. Alarcón JA, Bastir M, Rosas A, Molero J. Chincup treatment modifies the mandibular shape in children with prognathism. Am J Orthod Dentofacial Orthop. 2011; 140:38-43. [PubMed: 21724085] 


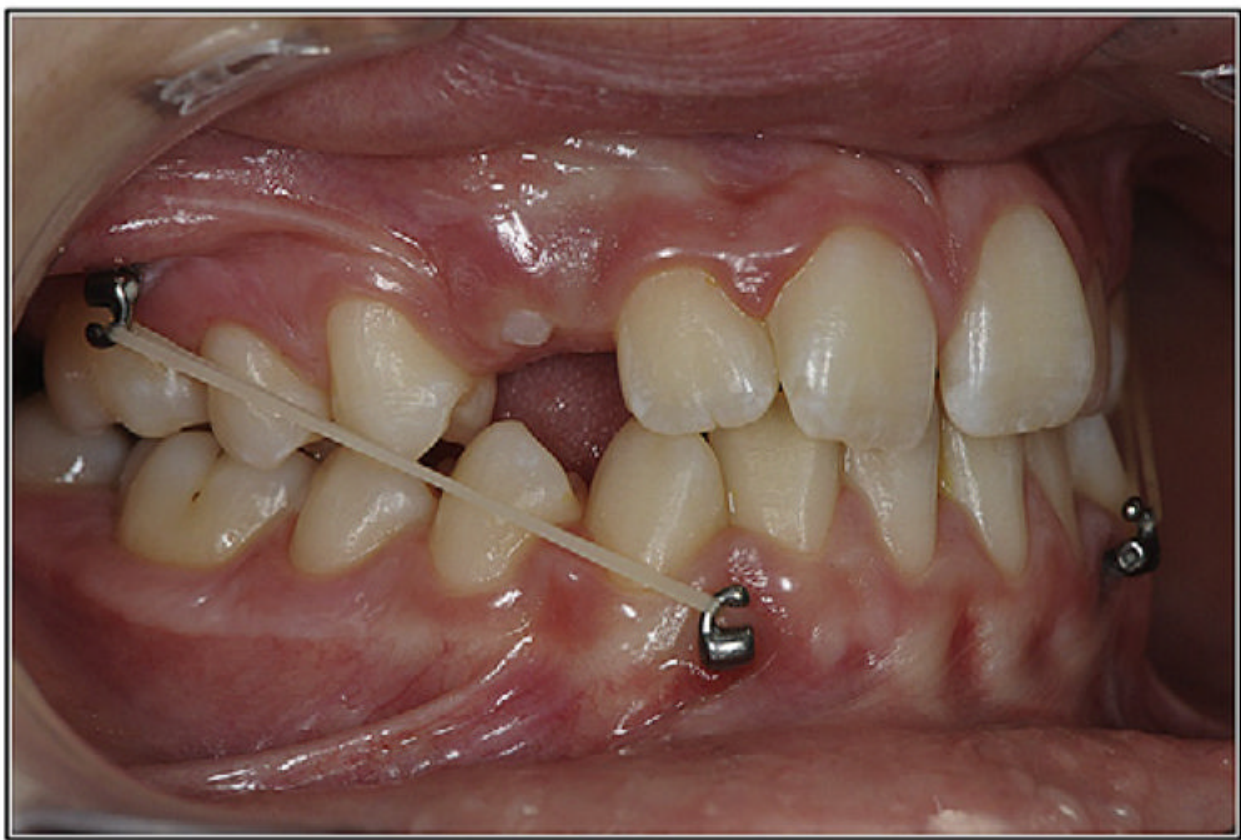

Fig 1.

Elastics are fixed for 24 hours a day between the extensions of the maxillary and mandibular miniplates. 


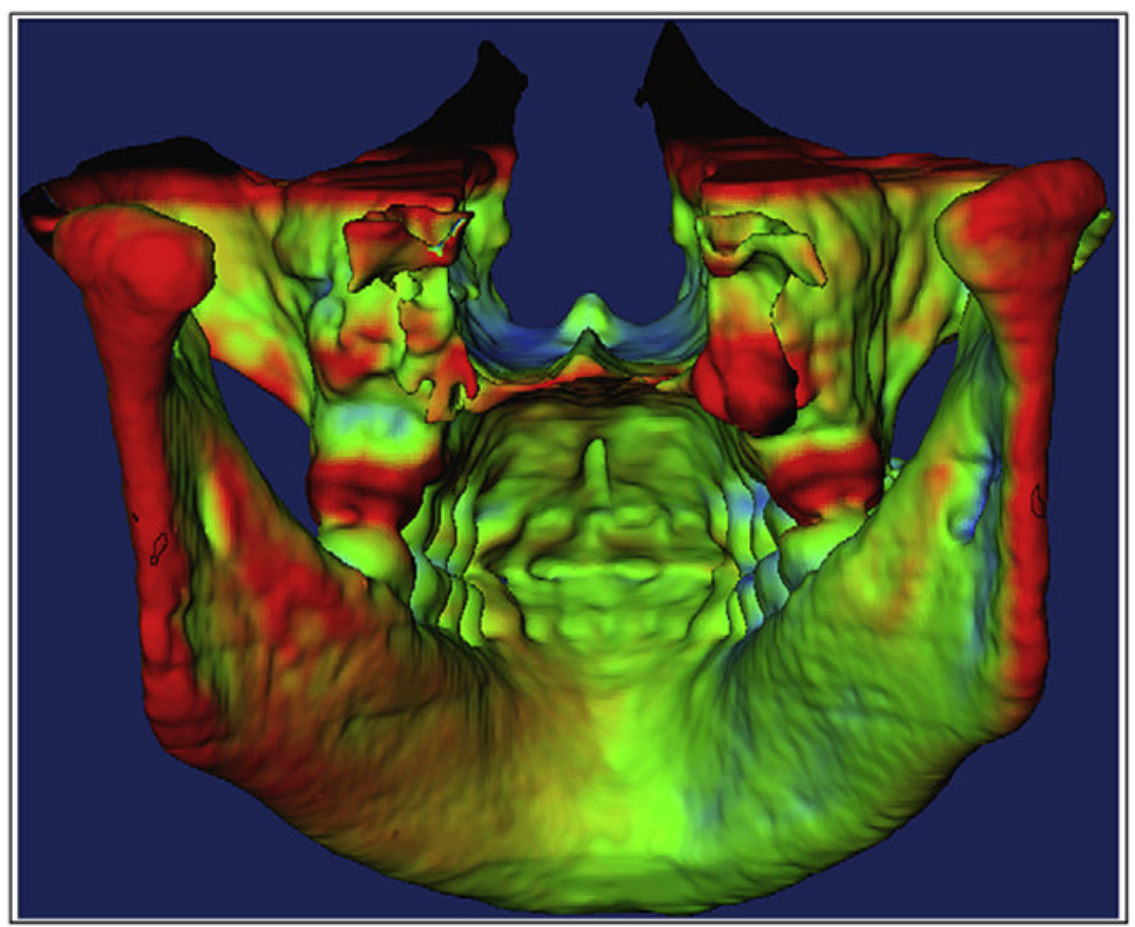

Fig 2.

Three-dimensional skeletal color map of the superimposition of the mandible registered on the anterior cranial base. The image of the isolines on the posterior border of the mandible shows regions with a displacement equal to or greater than a preset value. The red color of the posterior border of the ramus corresponds to a posterior displacement of $4 \mathrm{~mm}$. 


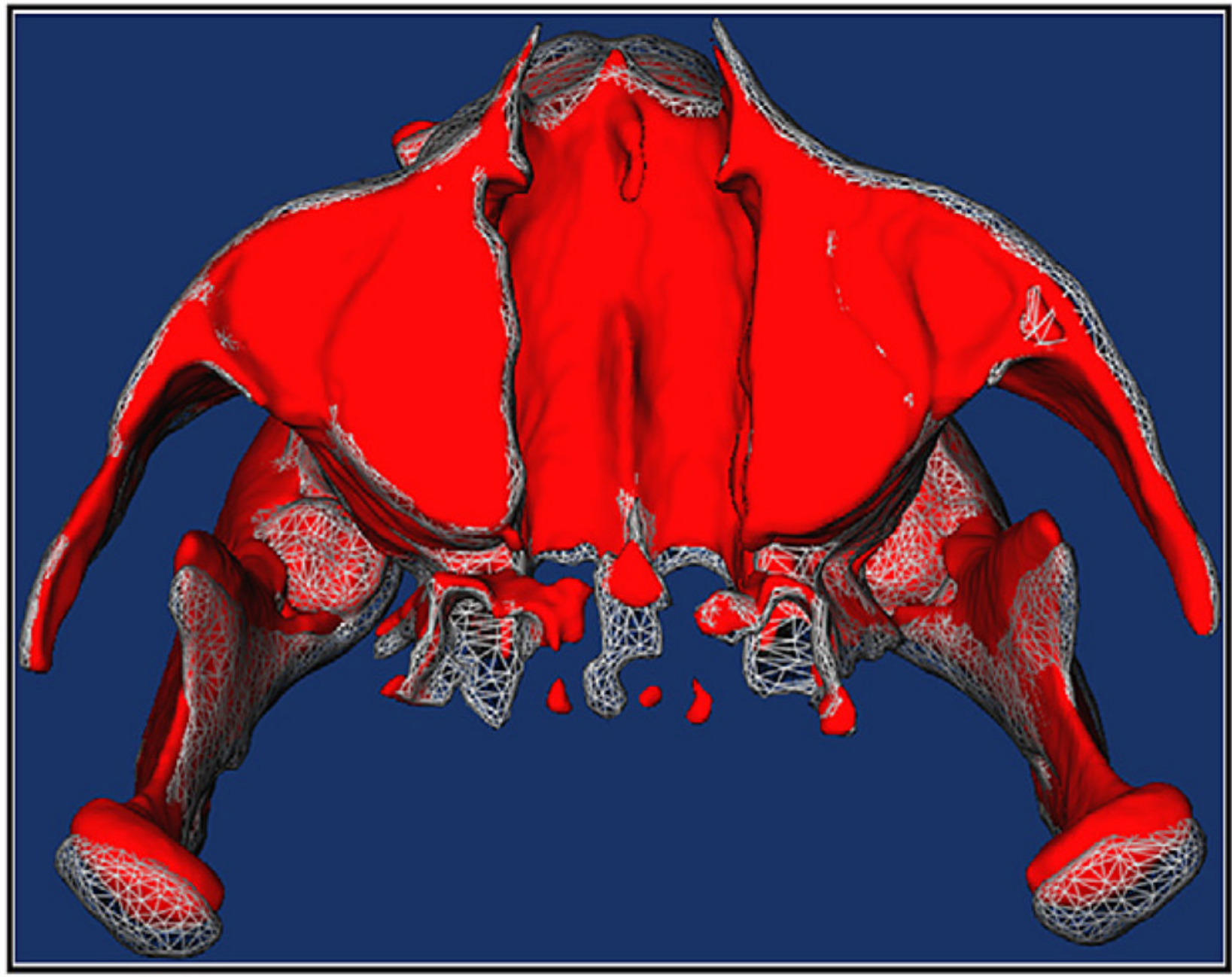

Fig 3.

Three-dimensional skeletal semitransparency superimposition registered on the anterior cranial base. The 3-dimensional image in red is from the CBCT image at T1, and the transparent overlay is from the CBCT image at T2 of the bone-anchored maxillary protraction protocol. The condyles moved posteriorly. 


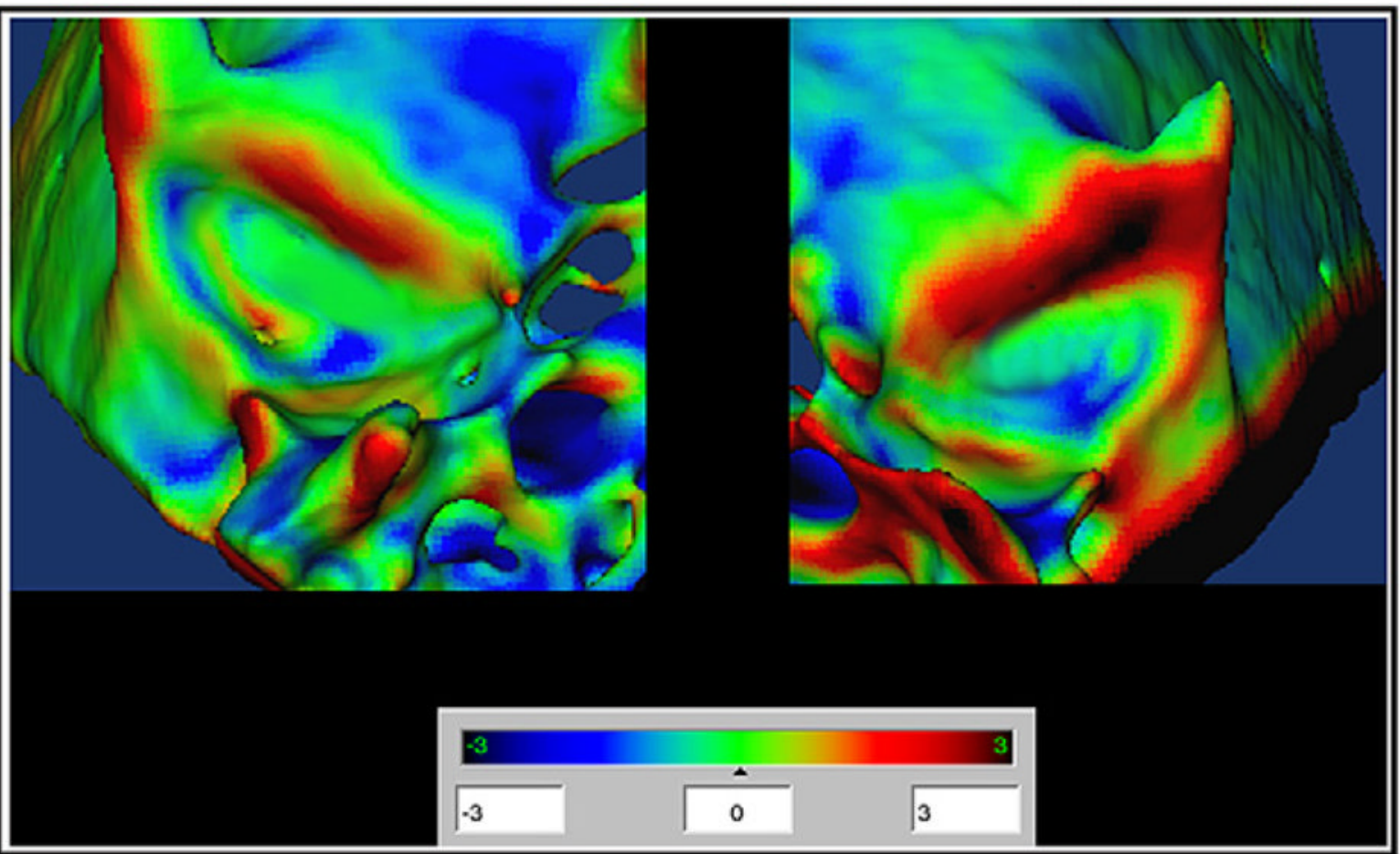

Fig 4.

Three-dimensional skeletal color map of the superimposition of the glenoid fossa at T1 over the image at T2 registered on the anterior cranial base with a scale of -3 to $+3 \mathrm{~mm}$ shows bone apposition at the anterior eminence (red) and resorption of the posterior wall (blue). The green color corresponds to surfaces without displacement. 

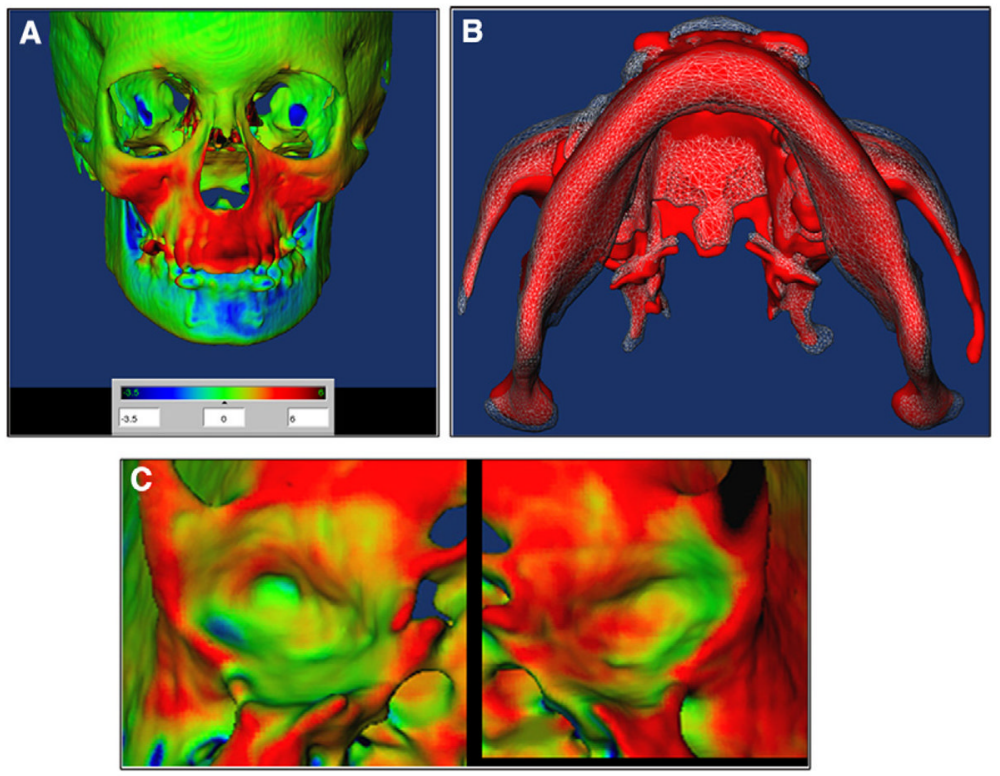

Fig 5.

A, Three-dimensional skeletal color map and $\mathbf{B}$, semitransparency superimposition registered on the anterior cranial base shows a slight asymmetric displacement of the chin to the patient's right side after unilateral elastic traction for about 5 months; $\mathbf{C}$, asymmetric movement of the condyles corresponds to asymmetric modeling of the left and right glenoid fossae. 


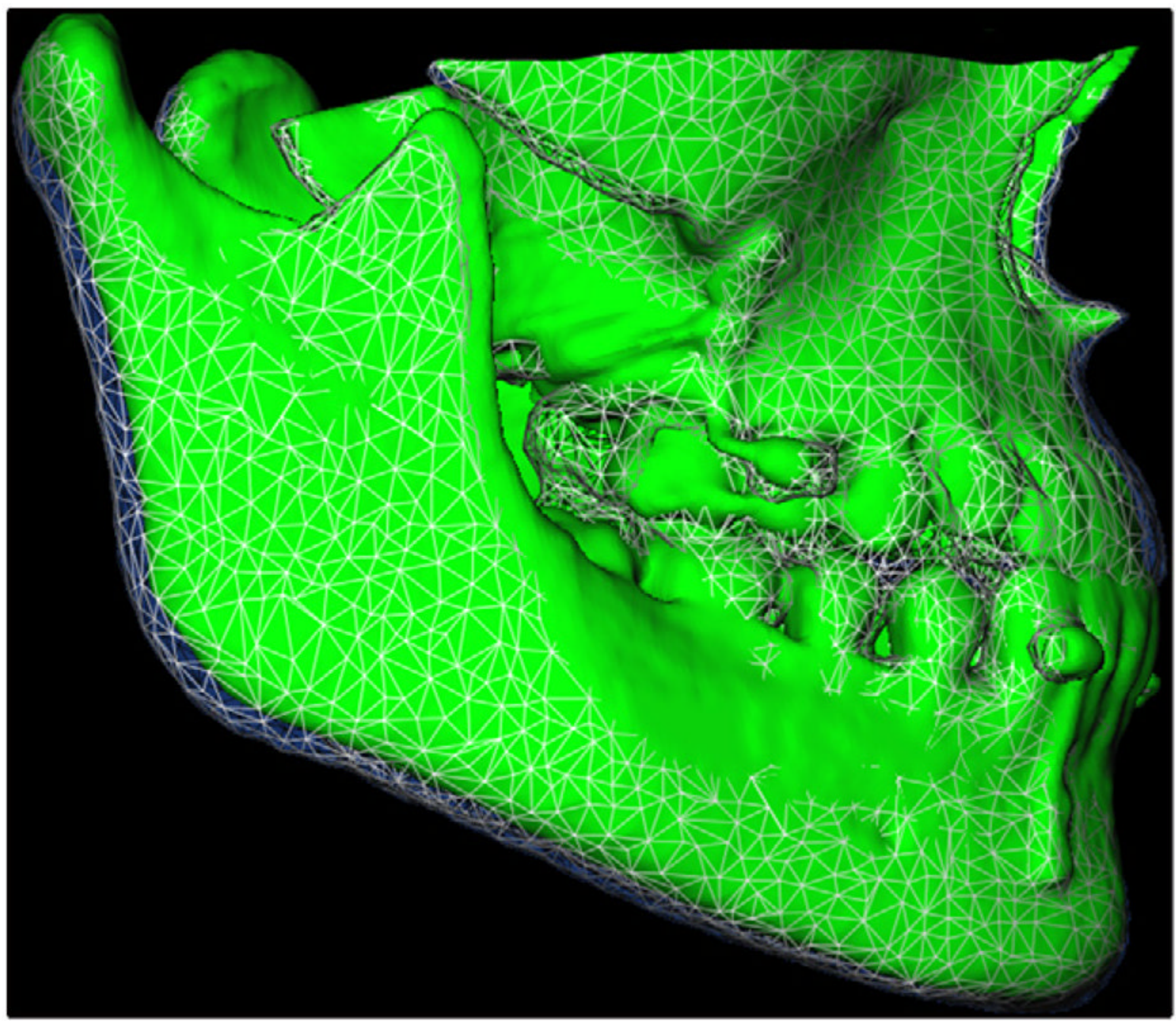

Fig 6.

Three-dimensional skeletal semitransparency superimposition registered on the anterior cranial base. The green volume is before treatment, and the transparent overlay is after treatment, illustrating a slight swing-back of the ramus and closure of the gonial angle with a small reduction of the mandibular plane angle. This results in restriction of the forward projection of the chin. 


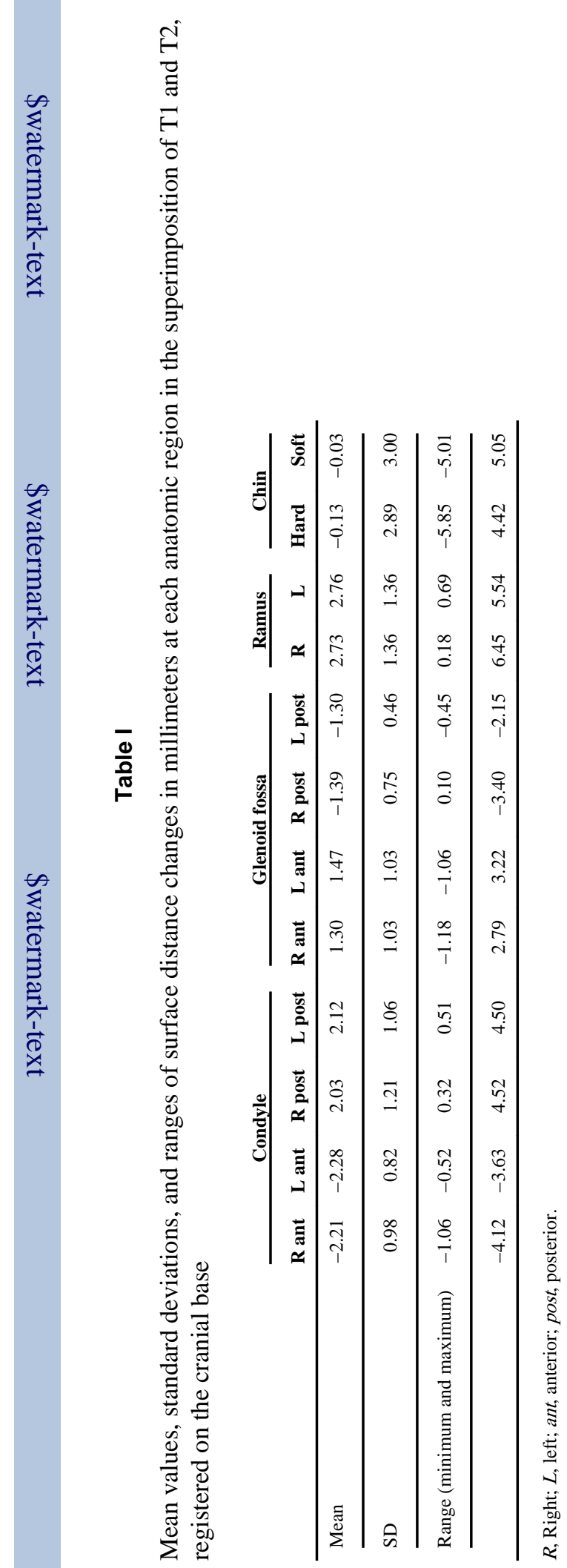




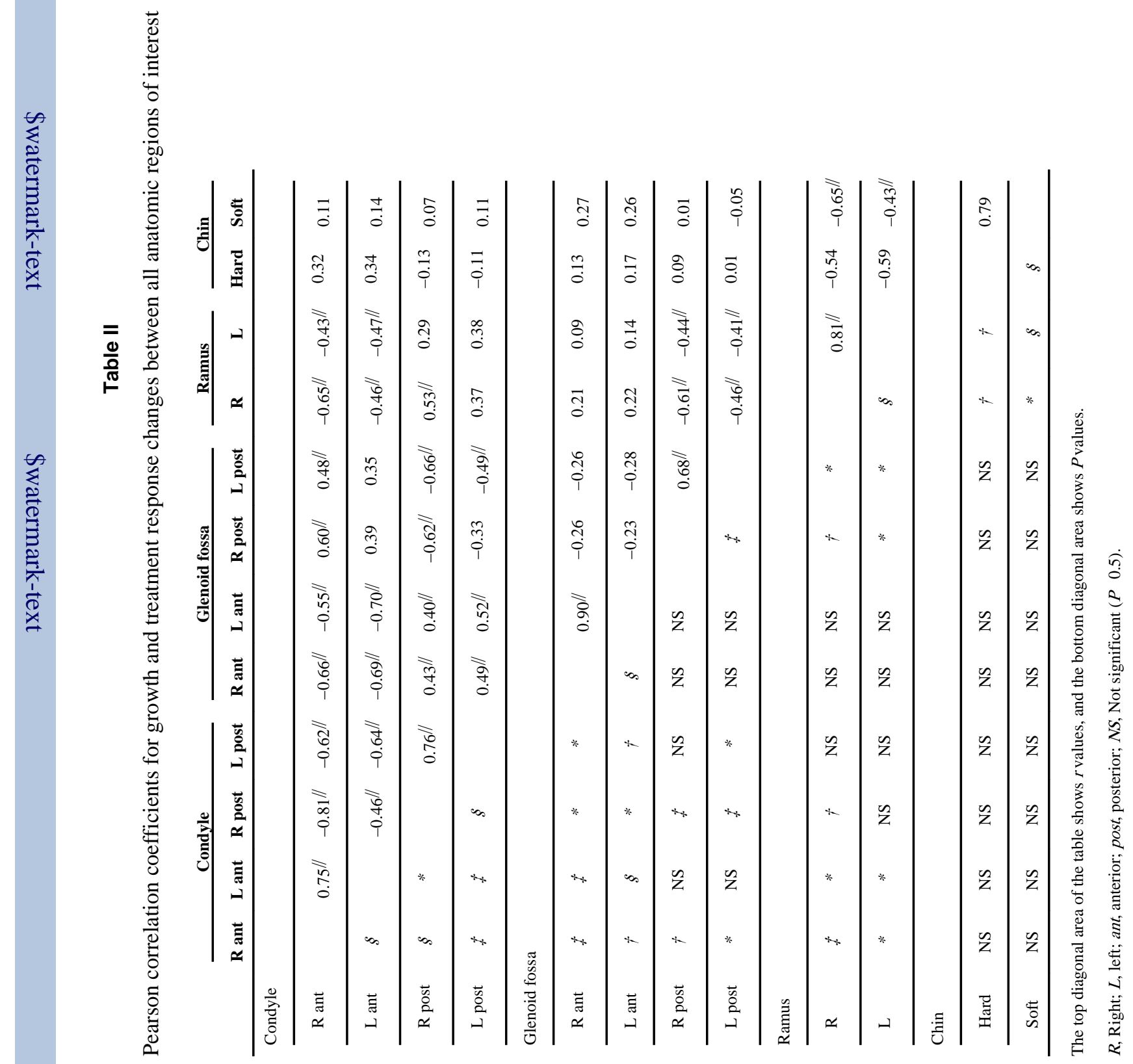




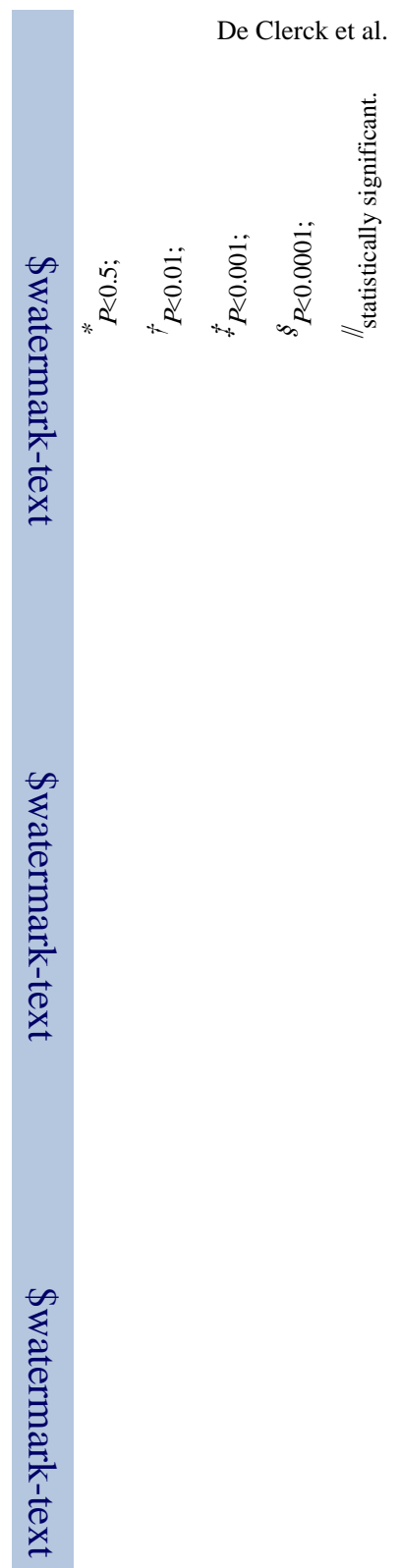

Am J Orthod Dentofacial Orthop. Author manuscript; available in PMC 2013 January 24. 